\title{
Concevoir des cadres pour faire et faire faire : l'activité d'encadrement dans une entreprise horticole
}

Design frameworks to make and do: management in a horticultural company

\section{Corinne Gotteland-Agostini, Valérie Pueyo et Pascal Béguin}

\section{(2) OpenEdition}

Journals

Édition électronique

URL : http://journals.openedition.org/activites/983

DOI : 10.4000/activites.983

ISSN : 1765-2723

Éditeur

ARPACT - Association Recherches et Pratiques sur les ACTivités

Référence électronique

Corinne Gotteland-Agostini, Valérie Pueyo et Pascal Béguin, «Concevoir des cadres pour faire et faire faire : l'activité d'encadrement dans une entreprise horticole», Activités [En ligne], 12-1 | Avril 2015, mis en ligne le 15 avril 2015, consulté le 19 avril 2019. URL : http://journals.openedition.org/activites/983 ; DOI : 10.4000/activites.983 


\title{
Concevoir des cadres pour faire et faire faire : l'activité d'encadrement dans une entreprise horticole Corinne Gotteland-Agostini
}

CMW (UMR 5283)

Université de Lumière Lyon 2

co.gotteland@gmail.com

\author{
Valérie Pueyo \\ CREA-EVS (UMR 5800) \\ Université Lumière Lyon 2 \\ valerie.pueyo@univ-lyon2.fr
}

\author{
Pascal Béguin \\ CREA-EVS (UMR 5800) \\ Université Lumière Lyon 2 \\ pascal.beguin@univ-lyon2.fr
}

\begin{abstract}
Design frameworks to make and do: management in a horticultural company. This text aims to produce knowledge about the work of supervisors, particularly in relation to production management: their productive mentoring activity. We analyze this activity as a design activity that produces frameworks of action for both doing the job and getting the job done. The research was conducted in a small horticultural company belonging to a national producers group which is itself made up of three regional groups. This structure defines in part the inner workings of the company. In addition, it requires not only frameworks for action which pre-exist the mentoring activity, but also external stakeholders with whom the managers must interact.

The analysis of observations and interviews shows that this production management activity develops through the running of daily and occasional microprojects, respectively led via co-framing and framing actions. The manager works alone, or with help from colleagues. Before giving orders, he/she has to organize production. This is achieved by confronting a constantly evolving work situation. The manager thus appears to be more than just a designer whose work is both framed and framing. He is a veritable project manager, responsible for synchronizing the work of a multiskilled team and for coordinating their work so as to achieve production and sales goals.
\end{abstract}

\section{KEYWORDS}

manager, frame to work, framing, production management, microproject 


\section{1.- Introduction}

\section{1.- Encadrants : catégorie, rôle et travail}

Parmi les encadrants, nous pouvons distinguer les encadrants de proximité. Gillet (2003) retrace l'histoire de cette catégorie de travailleurs qu'elle décrit comme extrêmement sensible aux changements organisationnels du fait de cette position intermédiaire qui les place entre l'enclume et le marteau (Mispelblom Beyer, 1996, 2003). Les écrits de Detchessahar (2011) dénoncent les conséquences du «management empêché » en termes de santé au travail. Cet auteur déplore le manque de présence des encadrants de proximités auprès de leurs subordonnés dans l'activité de travail, expliquant qu'ils sont trop occupés à alimenter les outils de gestion ou trop accaparés par tout un ensemble de groupes de travail découlant du développement du management participatif. Ce même auteur plaide pour un management de la discussion. D'après lui, «la santé au travail dépend de la qualité des dynamiques communicationnelles autour du travail, vu sous l'angle de ses conditions réelles de réalisation » (Detchessahar, 2013). Autre fait évoqué, les encadrants sont de plus en plus dépendants à l'égard du travail de collègues, avec des exigences de coordination élargies audelà du strict contour de leur équipe (Wolff, 2013). Au-delà de dimensions d'animation ou de gestionnaires, leur position «en première ligne » les amène à jouer un rôle d'intégration de l'ensemble du système de production (Gotteland-Agostini, 2013). Cependant, les représentations sociales dominantes tendent à les assimiler à de simples organes de transmission de consignes et de contrôle des subordonnés, alors qu'ils devraient plutôt être considérés comme des acteurs participant aux évolutions de l'entreprise (Létondal, 1997).

Depuis une vingtaine d'années, le travail des encadrants est devenu un objet d'analyse en ergonomie de langue française. Plusieurs recherches se sont attachées à montrer les spécificités de l'activité de ces travailleurs dans de grandes entreprises (Langa, 1994; Mascia, 1994, 2001). D'autres ont été, pour une partie, inspirés de la sociologie. Ces dernières se sont alors focalisées sur les régulations collectives (Carballeda, 1997a). L'encadrant y apparaît alors comme un opérateur aux conditions de travail spécifiques, à l'interface de contraintes ascendantes et descendantes du fait de sa position hiérarchique intermédiaire dans l'entreprise. Dans le BTP, des auteurs se sont intéressés aux aspects organisationnels de la production (Béguin \& Bergamini, 1996 ; Bergamini, 1995); d'autres ont montré des évolutions des métiers en lien avec la gestion des procédures (Six \& Tracz, 1997) ; d'autres encore ont analysé les conditions d'élaboration de la prescription visant à améliorer la prévention des risques (Six, 1999). Plus récemment, des études centrées sur les dimensions cognitives de l'activité des encadrants dans l'aéronautique (Lebris, 2010) et les Travaux Publics (Forrière et al., 2011), témoignent de l'intérêt croissant des ergonomes à l'égard de ces travailleurs. En horticulture, Zara-Meylan (2012) a démontré que leur activité consiste à gérer de l'incertitude et des contraintes temporelles multiples. Indépendamment de leur statut et de leur fonction, l'ensemble de ces travaux souligne la complexité de leur travail au regard des tâches multiples et variées qu'ils ont à accomplir: relationnelles, gestionnaires, techniques et commerciales (Mascia, 2001). D'autres études enfin évoquent les enjeux relevés au quotidien par des encadrants confrontés au cadre contraint du pilotage de la performance (Piney et al., 2013) ou à la nécessité d'un cadre formel d'exercice de la confiance (Van Belleghem, 2013). La littérature ergonomique étant peu abondante quant au travail des encadrants de proximité, nous nous sommes intéressés aussi au travail des cadres de manière à enrichir nos apports théoriques.

\section{2.- Poser des cadres : la spécificité du travail d'encadrement}

Les encadrants assurent avant tout un rôle de production et de maintient de l'organisation (Carballeda, 1997a). Le travail qui leur incombe les met parfois en difficulté, et peut même engendrer des problèmes de santé (Carballeda, 1997b ; Carballeda \& Garrigou, 2001). Cependant, force est de constater que leur travail demeure assez mal connu bien qu'il nous 
semble être doublement déterminant: pour comprendre non seulement leurs propres conditions de travail, mais aussi celles des personnels placés sous leur responsabilité. Peu de travaux décrivent effectivement ce qui constitue la spécificité même de leur activité : le fait d'encadrer. D'après Mispelblom Beyer (1996, 2003) «encadrer consiste à (im)-poser des cadres aux personnes encadrées : des limites à ne pas dépasser, une orientation de travail ». Alors, au-delà des apports que nous venons de citer, comment les encadrants s'y prennent-ils concrètement pour définir et prescrire le travail à faire et à faire faire ?

D'autant que comme le soulignent Castejon et Jean (1998), la faible prescription dont ils disposent pour eux-mêmes se résume souvent à : «débrouillez-vous pour atteindre vos résultats ». Cette imprécision des tâches à réaliser laisse une grande place dans leur activité à l'auto-prescription et à l'auto-organisation (Dieumegard, Saury, \& Durand, 2004 ; Rogard \& Béguin, 1997). De Terssac \& Cambon (1998) s'intéressent au «travail d'encadrement »dont l'objet est à envisager, selon eux, du point de vue d'une double activité consistant d'un côté à « créer les cadres » de l'action future, et de l'autre à « faire cadrer» les actions et à agir en sorte qu'elles ne sortent pas de ces cadres. Ainsi, d'après ces auteurs, toute la difficulté de leur activité consiste à délimiter le cadre, puis à faire cadrer pour que d'autres agissent de façon efficace et coordonnée ou pour «faire faire les choses» de manière à ce qu'elles se déroulent dans les cadres qui ont été préalablement établis.

\section{3.- Produire des connaissances sur l'encadrement productif}

Cet article a pour but de produire des connaissances sur le travail des encadrants. Nous n'avons cependant pas prétention à couvrir tout le champ du travail d'encadrement. Bien que nous ayons observé leur activité de travail dans sa globalité, nous focaliserons notre étude ${ }^{1}$ sur «l'activité d'encadrement productif » développée par l'encadrant dans l'exécution des tâches accomplies de gestion de la production, celles-ci renvoyant à l'organisation du travail de production, à l'élaboration de la prescription, ainsi qu'à la coordination et au suivi du travail des autres.

Pour analyser cette activité d'encadrement productif, nous nous appuierons sur une recherche menée sur l'activité d'un encadrant de proximité d'une petite entreprise horticole ${ }^{2}$. Comme nous le verrons, le fait de devoir prescrire le travail de production nécessite parfois au préalable, pour cette personne, de définir et d'organiser celui-ci en amont, alors même qu'il est en prise avec le réel et la dynamique de la situation de travail. Et pour ce faire, nous nous référerons à deux cadres théoriques structurants: la conception et de la conduite de projet (Béguin, 2010) ainsi que la sociologie interactionniste symbolique de Goffman (1974).

\footnotetext{
Cette recherche se devait de répondre à une double demande : i) aux préoccupations du projet de recherche au sein duquel cette étude a été menée, et plus particulièrement en lien avec les questions des préventeurs quant aux possibilités de prescrire et aux caractéristiques de la prescription du travail horticole ; ii) à la demande initiale de l'entreprise portant sur une problématique de qualité liée à la prescription du travail des ouvriers de production.

2 L'encadrant observé est responsable du secteur de production et expédition des commandes. Il est positionné hiérarchiquement entre la direction (deux co-gérants, un gestionnaire et un responsable du disponible) et les ouvriers de production, et au même niveau que le chef de culture. Il ne dispose d'aucun outil de gestion. L'affectation aux différentes tâches (chantiers et commandes) qui se fait en prise directe avec le réel de la situation (avancement du travail, arrivée de commande...), dans le face à face avec les ouvrier, et par voix orale (avec parfois une note manuscrite transmise en même temps).
} 


\section{2.- L'activité d'encadrement : une activité de conception de cadres d'action}

\section{1.- Une activité de conception vue sous l'angle de la conduite de projet}

De notre point de vue, l'activité d'encadrement peut être comprise comme une activité de conception grâce à laquelle l'encadrant conçoit des «cadres d'action » (Gotteland-Agostini, 2013).

Dans ce qui suit, nous envisagerons l'activité de conception au sens de Schön (1983) : l'activité réflexive d'un praticien qui «dialogue » avec la situation qui lui résiste et lui répond. Et nous appréhenderons cette activité de conception par la conduite de projet définie par Staudenmaïer (1985) comme étant une activité individuelle et collective, d'élaboration et de validation de modèles sous le contrôle d'un but. Envisagée sous cet angle, l'activité de conception est un acte maîtrisé et ordonné de conception qui poursuit un mobile (Béguin, Ibid). Ce concept renvoie à l'organisation du projet en tant que processus de conception. Ses principales caractéristiques résident dans le fait qu'il ne se modélise pas a priori, et qu'il implique la participation des acteurs aux compétences multiples (équipes pluridisciplinaires), à une grande échelle temporelle et organisationnelle avec de l'incertitude et des stratégies de conception diverses.

Nous retiendrons le modèle de la conduite de projet proposée par Béguin (2010) qui présente la conduite de projet comme (1) un processus finalisé, (2) aux dimensions temporelles contraintes avec de l'irréversibilité, (3) et qui met aux prises une diversité d'acteurs en interdépendance. Selon cet auteur, la conception est un processus qui s'élabore progressivement et continument jusqu'à atteindre le résultat final. Ce processus se déroule sur deux plans qui font référence à des temps différents.

Le premier plan part de l'activité de construction d'une finalité, d'une idée directrice, puis passe par la production d'ébauches multiples et aboutit à la réalisation d'une œuvre, d'un produit ou d'une situation de travail (Béguin, 2010). Le second plan consiste en une série de mises en tension entre des couples de pôles, qu'il s'agit de faire converger vers le concret, ce qui permet d'assurer cette transformation. Dans son élaboration, cette concrétisation va rencontrer des résistances émergeant du réel et de la situation de travail : «dans la conduite de projet, le virtuel, la représentation, contribue à mettre en forme un réel, mais dont les résistances et les possibles ne manqueront pas d'interroger, de remettre en mouvement les représentations initiales » (Béguin, 2010, p. 50).

Pour mieux comprendre cette dialectique à l'œuvre dans le processus de conception, nous nous référons à la théorie de l'agir développée par Joas (2004). Ce philosophe qualifie la pensée occidentale de «téléologique»: selon cette orientation, l'agir humain est compris comme la poursuite de fins préconçues qui sont ensuite déclinées en action. D'après lui, dans l'interprétation téléologique de l'intentionnalité de l'agir, il y a une dissociation entre la connaissance et l'action. La première est utile pour établir le plan préalable à la seconde. Pour agir, le sujet va construire un plan, car il dispose de toutes les ressources en lui et il agit sur l'environnement. Et accomplir l'action consiste à faire en sorte d'atteindre le but connu et fixé à l'avance, et ce, quels que soient les aléas rencontrés dans la réalisation de l'action. Selon cette pensée, le sujet est guidé par son mobile et son plan, sans que ceux-ci soient remis en cause dans la confrontation à la réalité à la situation. Ici, ce sont les structures cognitives du sujet qui lui permettent d'agir quelle que soit la situation.

En revanche, selon la pensée non téléologique de l'agir, il existe des ressources qui émergent de la situation et à partir desquelles le sujet va pouvoir agir en fonction de ses compétences. « Nous ne percevons une situation qu'en fonction de nos aptitudes et de nos dispositions actuelles à agir » (Joas, Ibid.). Ce même auteur précise que l'agir s'inscrit dans un rapport préréflexif avec la situation et il «ne présuppose plus nécessairement une quelconque 
planification ». Une conception non téléologique de la relation entre l'agir et la situation du sujet serait une conception «quasi dialogique » (Böhler, 1985), ce qui signifie que les actes doivent être compris comme des réponses à des situations. En effet, «les situations ne sont pas muettes, elles appellent les actes » même si elles ne les provoquent pas et qu'elles ne représentent pas non plus le simple arrière-plan sur lequel nous réalisons nos intentions (Joas, Ibid., p. 171). Ici, le problème se construit «en avançant», suivant le déroulement de l'action et dans la confrontation au réel.

Ainsi, pour créer ou concevoir, le sujet s'appuie sur les ressources que lui propose la situation, qu'il interprète et identifie comme telles en fonction de ses connaissances et de ses compétences.

\section{2.- Des cadres primaires transformés ou des cadres secondaires : les cadres d'action}

Pour tenter de comprendre ce travail d'interprétation de la situation, nous nous appuierons sur le concept de cadre tel qu'il a été développé par Goffman ${ }^{3}$ (1974) dans son dernier ouvrage «Les cadres de l'expérience». Ce sociologue s'inspire de la métaphore cinématographique : le cours de l'existence est composé de multiples «constructions de la réalité » que chacun opère au moyen de différents « cadres » («frames ») s'articulant les uns aux autres et qui forment l'expérience. Il considère que pour identifier un évènement et agir de manière appropriée, le sujet fait appel à des cadres primaires, qui lui permettent de comprendre «ce qui se passe ici ?».

Toute expérience, toute activité sociale se prête d'après lui à plusieurs versions ou cadrages. Entretenant des rapports les uns avec les autres, les cadres fixent la représentation de la réalité, orientent la perception et influencent l'engagement et les conduites de chaque individu dans sa relation au monde et aux autres. Passant inaperçus, ils sont néanmoins partagés par toutes les personnes en présence. Ici, le cadre structure l'interaction et définit la situation. Il n'appartient pas en propre aux personnes qui interagissent, mais à la situation. Ce qui appartient en propre aux personnes c'est la modalisation de l'expérience (la perception de la situation) qui fait qu'elles utilisent le bon cadrage pour comprendre ce qui se passe et se joue dans cette situation et qui leur permet d'agir ensemble de manière adéquate.

Goffman (Ibid.) distingue les «cadres primaires» des «cadres secondaires». Est primaire un cadre qui nous permet, dans une situation donnée, d'accorder un sens à tel ou tel de ses aspects, lequel autrement serait dépourvu de signification »(p.30). Le cadre secondaire est quant à lui un cadre primaire transformé. «On a affaire à un cadre transformé lorsque la situation, bien que présentant certaines ressemblances avec le cadre primaire, acquiert pourtant une signification différente» (Nizet \& Rigaux, 2005). Les cadres secondaires peuvent subir plusieurs degrés de transformation. "L'expérience peut ainsi être décomposée en autant de "strates", combinant modalisations et transformations » (Nizet \& Rigaux, op.cit.).

De plus, Goffman identifie deux grandes classes de cadres primaires : les «cadres naturels » et les «cadres sociaux». Les cadres naturels «nous permettent d'identifier les occurrences qui ne sont pas ordonnées ou orientées, qui ne sont pas pilotées ou animées, qui sont «purement physiques» (p. 30). Ces occurrences ne répondent à aucune évaluation

\footnotetext{
3 «Bien que Goffman emprunte la notion de cadre à Bateson, il lui donne une signification différente. Les cadres ne résultent plus, chez lui, d'un genre de convention qui s'établirait entre les individus en communication. Ils sont devenus indépendants des intentions individuelles ; ils constituent des objets d'analyse en tant que tel, qu'il est possible de classer, d'articuler les uns aux autres » (Sharron, 1981/2000, p. 97-98).

4 En italique dans le texte original.
} 
particulière de notre part. Ces cadres naturels renvoient en autres à l'élément naturel, aux conditions climatiques et météorologiques. Quant aux «cadres sociaux », ils «permettent de comprendre d'autres évènements, animés par une volonté ou un objectif et qui requièrent la maîtrise d'une intelligence; ils impliquent (...) l'agent humain.(...) [celui-ci] doit prêter attention à toute une série de choses qui portent à conséquence, assurer un contrôle correctif, continu, veiller que l'action ne soit pas bloquée ou déviée de manière inattendue ». Un élément important relatif à ce concept de cadre social réside dans le fait que « pris ensemble, les cadres primaires d'un groupe social constituent l'élément central de sa culture ». Vues sous cet angle, l'organisation du travail, les règles ainsi que les prescriptions font partie des cadres sociaux.

Par ailleurs, il arrive que l'interprétation faite des cadres primaires soit erronée par rapport à la réalité à laquelle un sujet se trouve confronté: «il nous arrive (...) de faire de "mauvaises" interprétations, c'est-à-dire d'être à côté de la plaque»(Goffman, Ibid., p. 35). L'auteur parle alors d' «erreur de cadrage » qui peut se produire dans le cas d'activités franches ou de fabrications, et à l'issue de laquelle l'individu réalise après un temps plus ou moins long, «qu'il a perçu la situation de manière erronée et qu'il s'y est engagé de façon inadéquate » (Nizet \& Rigaux, Ibid. p. 71). Goffman (Ibid.) appelle cela une « rupture » de cadre.

Nizet et Rigaux (2005, p. 72) soulignent la double fonction de ces cadres : d'abord, en orientant les perceptions et les représentations ${ }^{5}$, ceux-ci « donnent à l'individu l'impression que cette réalité est bien ce qu'elle est». Ensuite, en influençant ses conduites, car «la définition de la réalité étant fixée, la personne peut ajuster son degré d'engagement et adopter des comportements adéquats ». C'est donc la perception de la réalité qu'adopte le sujet qui va le motiver à agir dans un sens plutôt qu'un autre. Sachant qu'un opérateur, dans son activité de travail, va agir en fonction d'éléments internes (de son état de santé, de sa propre histoire et ses compétences) et externes à lui-même (composants de la situation de travail).

Grossen (2001) précise quant à elle la différence entre le terme de «cadre » et celui de « cadrage ». Alors que le premier met l'accent sur « les règles qui structurent toute situation et en font un évènement au moins partiellement identifiable»; le second renvoie à « l'activité d'interprétation du sujet et sa capacité de transformer les règles du cadre par son activité même» (p. 61). Le travail de cadrage est un travail de modalisation de l'expérience qui consiste à conférer un sens autre à la situation et qui aboutit à la conception d'un nouveau cadre pour définir une situation donnée.

Ainsi, les cadres primaires sont des schèmes interprétatifs qui nous permettent d'agir et de concevoir en lien avec la situation et en interaction avec les autres. Nous considérons que ces cadres primaires constituent des ressources pour l'encadrement (et tout autre sujet) pour et dans l'action. Cependant, bien que ceux-ci se trouvent en amont de l'action, ils n'ont pas pour fonction d'anticiper ou de planifier l'action à mener comme le sont les représentations pour l'action de Béguin et Weil-Fassina (1997).

Mais cela laisse à penser que lorsque la situation évolue, les cadres bougent, entraînant une réinterprétation dans l'action de la part du sujet, pour actualiser sa représentation de la réalité. Comme le montrent les observations de Schön (1983), dans la pratique, les concepteurs font souvent face à des situations nouvelles qui résistent à l'application des connaissances et des techniques scientifiques. Et quand ils sont confrontés à ces résistances du réel, c'est leur capacité à réfléchir dans et sur l'action qui leur permet de trouver des idées originales pour faire face à ces situations particulières, voire uniques. Ces concepteurs

Représentation au sens où l'entend l'interactionnisme symbolique, c'est-à-dire « une perception du monde, de la réalité ou de la situation » (Becker, 1963). C'est bien à cette référence situationnelle que nous faisons référence dans notre travail, en tant que manière d'interpréter la réalité quotidienne du travail. 
entrent alors « en dialogue avec la situation » pour "produire» des représentations d'un artefact. Grâce à ces conversations avec la situation, ils (re)travaillent les cadres en fonction des problèmes au fur et à mesure de l'avancement de leur réflexion et des réponses que leur renvoie la situation (Schön, op.cit.).

Ainsi, l'encadrant rencontre des contingences situationnelles: des résistances qu'il doit prendre en compte ou au contraire, des opportunités qu'il peut saisir pour agir et qui le conduisent à transformer les cadres primaires naturels et sociaux en cadres secondaires (cadres primaires transformés). Par son activité d'encadrement, il participe à l'élaboration des cadres primaires sociaux. En effet, il interprète les cadres primaires naturels et sociaux qu'il transforme par des actions de cadrage en une succession de cadres secondaires qu'il conçoit et transmet aux ouvriers qui vont à leur tour interpréter comme des cadres primaires sociaux sur lesquels ils vont s'appuyer pour répondre de manière adéquate à la demande de leur supérieur hiérarchique.

Nous appellerons «cadres pour l'action», les cadres primaires (naturels et sociaux) qui préexistent au travail de l'encadrant et «cadres d'action » les cadres secondaires conçus par l'encadrant et destinés, entre autres, à cadrer le travail des équipes sous sa responsabilité. L'objectif de cette communication est donc d'analyser l'activité de conception des cadres d'action produits par un encadrant du secteur horticole.

\section{3.- Terrain et méthode}

\section{1.- Des cadres primaires dans une entreprise du secteur horticole}

L'entreprise dans laquelle s'est déroulée la recherche compte une vingtaine de travailleurs permanents et autant de saisonniers durant la pleine saison (de mars à juin). Sur cette période, les travaux de production se cumulent avec les ventes de plantes vivaces ${ }^{6}$, de zonales $^{7}$ et d'annuelles. Le marché est axé uniquement sur la vente en jardineries. De ce fait, aucun client n'est présent sur l'exploitation.

Trois cadres primaires de cette situation sont déterminants pour l'activité des encadrants :

- La production est réalisée dans un environnement dynamique et ouvert, caractéristique du secteur horticole (Zara-Meylan, 2012). L'incertitude liée à l'élément naturel est omniprésente et regroupe : les conditions climatiques et météorologiques; les plantes considérées comme des produits vivants dont la croissante et la qualité évoluent sans cesse ; les règles culturales, botaniques et sanitaires, nécessaires pour arriver à produire des végétaux sains et de qualité.

— L'entreprise appartient à un groupement national de producteurs composé de trois groupements régionaux. Le niveau national gère les aspects marketing, les catalogues produits, facilitant aussi les achats groupés de matériel et d'intrants. Le niveau régional intervient en temps de vente. Son service commercial sert d'intermédiaire entre l'entreprise et les clients, il transmet les commandes passées par ces derniers aux différents producteurs. Il gère le dispatch de la plateforme logistique. Cette organisation en groupement confère à l'entreprise une latitude décisionnelle limitée, lui imposant des règles hétéronomes (Petit, 2005), élaborées en externe à chaque niveau du groupement. Cette première catégorie de règles conditionne la production de règles endogènes (Petit, op. cit.) en interne, permettant ainsi à l'entreprise de se structurer et de s'organiser pour fonctionner au sein du groupement.

- L'organisation de la production dictée par le calendrier des ventes du groupement implique que durant la pleine saison, le travail au secteur de la production et des ventes

6 Plantes dont les racines vivent au-delà de deux ans, en opposition aux plantes annuelles ou bisannuelles.

7 Variété de géranium. 
alterne entre des jours de commandes, des jours de production et des jours mixtes (combinaison des deux premiers).

L'ensemble de ces caractéristiques représente des cadres primaires en partie constitutifs des cadres pour l'action situés en amont du travail de l'encadrant. Ils représentent des ressources sur lesquelles celui-ci va s'appuyer pour concevoir les cadres d'action destinés à ses subordonnés.

\section{2.- Méthode d'une analyse de l'activité de conception de cadres d'action de l'encadrant}

Pour comprendre l'activité de conception des cadres d'action, nous avons centré nos observations sur l'activité de l'encadrant chargé, dans cette entreprise horticole, du secteur de la production et de la vente. Celui-ci organise, prescrit et coordonne le travail de production et de préparation de commandes des ouvriers. Nous avons procédé par étapes, en utilisant diverses approches.

Nous avons tout d'abord repéré les cadres primaires préexistants (naturels et sociaux) émanant du groupement et de la structure. Pour ce faire, nous avons examiné les documents organisationnels formalisés par l'entreprise (organigramme de l'entreprise, charte qualité du groupement régional, catalogue des produits et calendrier de vente du groupement national). Pour l'analyse, ces documents ont été associés à des entretiens auprès du personnel : les codirigeants, le chef de culture, l'encadrant du secteur de la production ainsi que quelques ouvriers (permanents et saisonniers).

Puis, nous nous sommes attachés à mettre en évidence, grâce à l'Analyse Ergonomique du Travail, les cadres secondaires élaborés dans l'action par l'encadrant. D'une part, ceux pour faire ou penser la situation, pour lui (avec le planning) et pour les autres (pour l'affectation des équipes), et ceux pour faire faire pour les autres (pour aider à la réalisation des travaux de production et des commandes).

Pour ce faire, nous l'avons observé dans une diversité de situations en lien avec l'organisation de la production, pour montrer une partie de la variabilité industrielle qu'il a à gérer :

— sur plusieurs jours d'un même type de fonctionnement de l'entreprise, c'est-à-dire, en jour de commandes, en jour de production et en jour mixte (production et commandes) ;

— sur plusieurs jours consécutifs d'une même semaine pour voir comment il procède pour passer d'un type de fonctionnement à un autre ainsi que d'une journée à l'autre, sachant que les trois types de journées ne se suivent pas, mais alternent.

Des observations ouvertes menées sur trois matinées consécutives de $8 \mathrm{~h}$ à $12 \mathrm{~h}$ nous ont permis de définir les observables pour analyser plus finement son activité d'encadrement. Ces trois demi-journées nous ont aidées à mettre en place le protocole des observations systématiques et à mettre au point la méthode pour suivre l'encadrant sur sa journée de travail (prise de notes, enregistrements audio...).

Les observations systématiques se sont déroulées sur onze journées complètes de $8 \mathrm{~h}$ à $17 \mathrm{~h}$. Sur la période observée, les effectifs sous ses ordres étaient composés de 12 permanents et de 14 saisonniers. En vue d'analyser son activité d'encadrement, nous avons noté au fil des heures ses actions, ses interlocuteurs, ses déplacements. Nous avons enregistré ses échanges verbaux avec chacun de ses collègues (hiérarchie et subordonnés) pour être en mesure d'identifier les cadres pour l'action auxquels il était confronté, les cadres secondaires transformés (dont cadres d'action) ainsi que les commentaires spontanés faits dans le cours de l'action, alors que l'encadrant préparait le travail (planning, notes manuscrites). Notre but était de recueillir des éléments sur les moments de transformation des cadres primaires (raison, forme, contenu, échanges). Nous avons ensuite retranscrit ces données, que nous avons rassemblées pour obtenir le déroulement de son activité sur chacune des journées observées. 
Ces retranscriptions ont servi pour conduire des entretiens semi-directifs en appuyant sur des traces de son activité (plannings, notes manuscrites, échanges verbaux) que nous avions recueillies lors des observations. Et nous avons mené des autoconfrontations (Mollo, 2004) sur 27 moments d'élaboration des cadres secondaires auprès des salariés, dont 12 en temps de production et 15 en temps de commandes. Parmi ces cas observés, 13 portaient sur la conception de cadres d'action identifiés (ceux qui nous intéressent), dont 7 sur des travaux de production et 6 sur de la préparation de commandes. Présentons sans plus attendre l'activité d'encadrement de cet encadrant.

\section{4.- Des cadres d'actions pour faire et pour faire faire}

\section{1.- Une conception de cadres d'action en trois étapes}

Comme nous l'avons écrit plus haut, le fait d'encadrer consiste à poser des cadres, autrement dire à prescrire puis à s'assurer que les tâches prescrites sont bien exécutées.

Deux résultats saillants ressortent de notre analyse. Le premier est relatif au fait que cet encadrant ne conçoit pas que de la prescription. Il conçoit d'abord de l'organisation du travail de production pour pouvoir ensuite prescrire le travail des ouvriers horticoles. Le second montre que pour prescrire, l'encadrant ne se contente pas de «transmettre tel quel» aux autres les cadres primaires sociaux qu'il reçoit, ou «d'adapter » pour lui-même ou pour les autres ceux qui lui sont adressés. Bien au contraire, il va aussi «concevoir» des cadres d'action pour faire (pour lui-même et pour les autres - ou organisation conçue) et faire faire (les autres - ou prescription conçue). Précisons que nous définissons la prescription comme un processus de conception qui aboutit à une action (Agostini-Gotteland, 2013). Or, qu'estce que concevoir de la prescription si nous considérons celle-ci comme un processus qui mène à une action?

Cette activité de conception s'opère en trois étapes (voir Figure 1, ci-dessous) : l'encadrant (1) définit l'action à réaliser, (2) il la cadre puis (3) il s'assure de sa réalisation. Cependant, la description de ces étapes ne doit pas laisser penser que les cadres d'action conçus sont stabilisés une fois pour toute car, comme nous le montrerons plus loin, dans la confrontation au réel ces cadres secondaires seront transformés à leur tour successivement par l'encadrant lui-même, avec les autres, comme nous le montrerons plus loin dans ce texte.

Ainsi, lors d'une première étape, l'encadrant définit les actions qui seront à faire et à faire faire. Pour y parvenir, il s'appuie sur les cadres primaires (naturels et sociaux) propres à l'entreprise au groupement de producteurs et du secteur. À ce stade, l'encadrant s'interroge sur le «pourquoi faire» tel ou tel travail? Et sur le «que faire» ou quels chantiers ou quelles commandes dois-je lancer? Autrement dit, quelles sont les actions et quelles sont les priorités?

Lors d'une seconde étape, l'encadrant cadre les actions. Il conçoit des cadres pour ses propres actions et celles des autres. Comment faire réaliser ces actions ? Comment affecter ? Comment organiser? Pour cela, il transforme les cadres primaires en cadres secondaires en s'appuyant sur les informations qui lui sont transmises, sur les éléments qu'il prélève dans la situation et sur les outils (planning de travaux) qu'il construit. Lors de cette phase, il répond d'abord au « comment faire pour faire faire? » ou comment organiser ce travail à partir des personnels et des moyens matériels dont il dispose ? Puis au «comment faire faire? » ou comment affecter les ouvriers en fonction de leur statut, de leurs compétences et des travaux à faire ? Cette étape aboutit à la conception d'une part, de cadres pour faire pour luimême et pour les autres, et d'autre part, de cadres pour faire faire destinés à ses subordonnés.

Lors d'une troisième étape, il s'agit de passer à la réalisation de l'action. L'encadrant poursuit donc la conception des cadres d'action dans la confrontation au réel, en fonction de l'avancement des travaux, en tenant compte des évolutions dans la situation de travail 
(salariés, végétaux, météo, etc.) et des différentes interactions avec les subordonnés, pairs, hiérarchie voire collègues du groupement. Il veille alors au «faire faire » en coordonnant le travail pour s'assurer de sa réalisation et au «faire» des ouvriers en charge de cette réalisation sur les chantiers ou lors de la préparation des commandes.

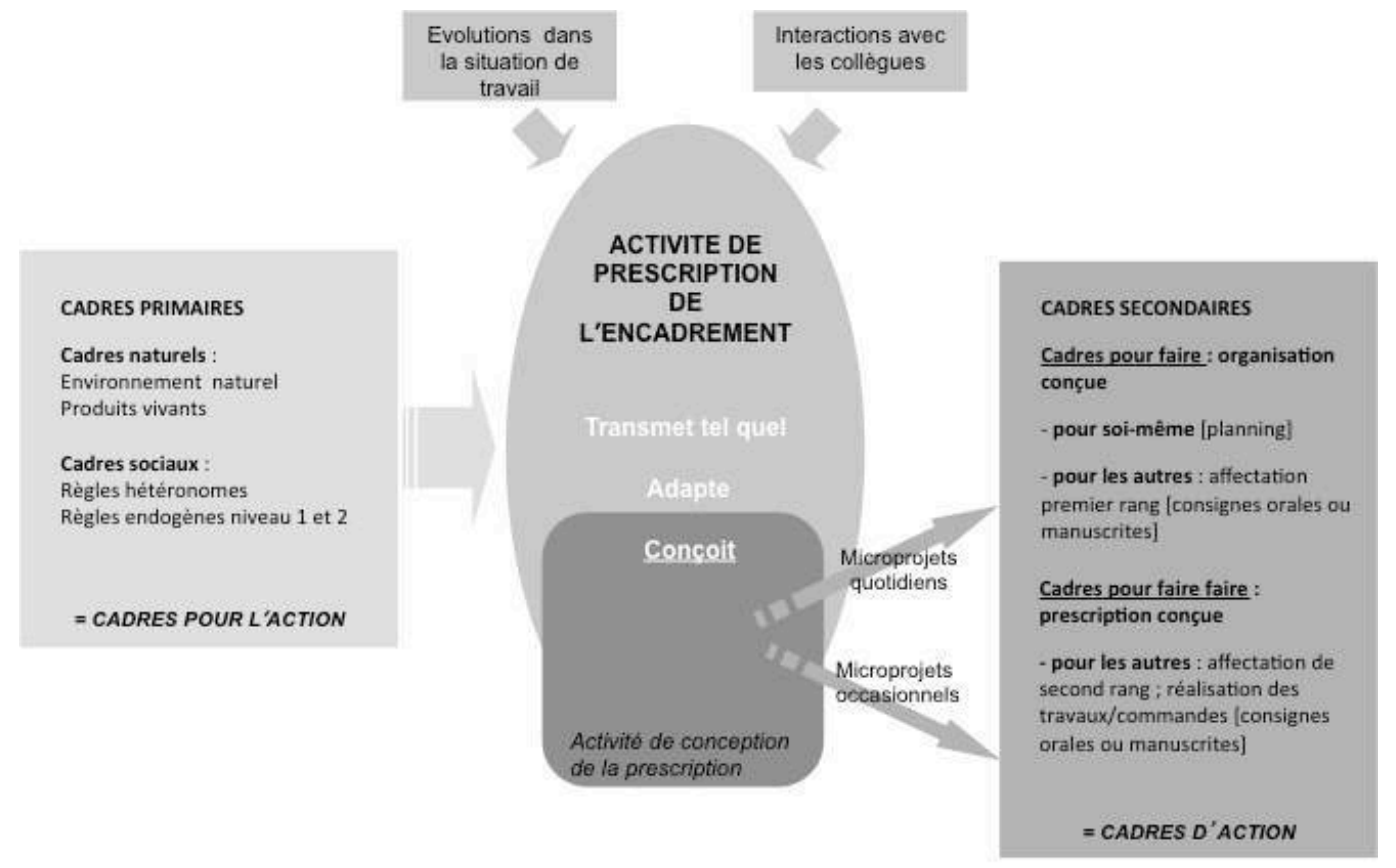

Figure 1 : L'activité de prescription de l'encadrement, concevoir des cadres secondaires dits cadres d'action

Figure 1: Managerial activity of giving orders, designing secondary frameworks (frameworks for action)

\section{2.- Une conception de cadres d'action inscrite dans des microprojets}

Comme nous l'avons évoqué précédemment, à l'aune des interactions, des variabilités industrielles, etc., l'encadrant va être conduit à transformer à plusieurs reprises les cadres d'action (cadres secondaires) pour lui-même et pour les autres, pour faire et pour faire faire. Cette remise en mouvement s'inscrit toujours dans des « microprojets » (Gotteland-Agostini, 2013). Ceux-ci peuvent être envisagés comme autant de projets développés par l'encadrant pour remplir ses missions autrement dit, pour accomplir les tâches d'encadrement qui lui incombent. Selon nous, ce dernier gère son activité de conception des cadres d'action en conduisant deux types de microprojets. Plus globalement, la notion de microprojet se caractérise par: une durée réduite (allant de quelques jours à quelques minutes), une conduite assurée par l'encadrant, produisant des actions de cadrage ou de co-cadrage et aboutissant à la conception de cadres d'action.

Le premier, que nous avons appelé «microprojet quotidien» répond à sa mission de tous les jours ayant pour but final de réussir à boucler «tout le travail à faire dans la journée ». Nous entendons par ces termes: i) les urgences qui sont des travaux de production à faire en priorité pour éviter de mettre en péril le végétal. Elles sont définies tous les matins dès leur arrivée sur l'exploitation par le directeur, le responsable du disponible ou le chef de culture ; ii) les travaux de production listés sur la feuille de travaux transmise par le chef de culture chaque semaine à l'encadrant; iii) les commandes transmises par le responsable de la saisie des commandes si c'est un jour de commandes. À partir de ces différentes données, l'encadrant va conduire seul et quotidiennement son microprojet en construisant des outils (i.e. planning de travaux) pour tenir ses objectifs de production et de vente. Il conduit un nouveau microprojet pour chaque journée de travail. Les cadrages sont produits lors de la 
conduite de ces microprojets quotidiens et uniquement par l'encadrant.

Le second, nommé «microprojet occasionnel », est une réponse à une mission ponctuelle assignée par sa hiérarchie. Ici, contrairement au cas précédent, l'objectif n'est pas de faire aboutir obligatoirement le processus de conception sur la même journée. Le tempo de ce projet varie en fonction du but désigné (i.e. la préparation d'une grosse commande une semaine à l'avance ou la manière de procéder pour poser des pots devant être arrosés par capillaires quelques secondes avant d'accomplir cette tâche). Dans ce cas, la contribution des collègues l'aide dans l'élaboration des solutions à apporter au cas par cas. Les co-cadrages sont réalisés au cours des microprojets occasionnels, en collaboration avec les collègues.

Voici à présent deux exemples de la conduite de ces microprojets, d'actions de cadrages et de co-cadrages permettant d'aboutir à la conception de cadres d'action pour faire et faire faire.

\section{3.- La conception de cadres secondaires pour faire dans un exemple de microprojet quotidien}

Le premier exemple concerne le microprojet quotidien du 27/5 (cf. Figure $2:$ « Le microprojet quotidien conduit par des cadrages successifs », située ci-après). Il illustre le mouvement de cadrages évoqué ci-avant. Pour le 27/5, prenant en compte les prévisions météorologiques annoncées par Météo France, l'encadrant a conçu son "planning de travaux ${ }^{8}$ » la veille en fin d'après-midi "en partant sur du beau temps ». Or, le matin en arrivant à $8 \mathrm{~h}$, il pleut. Certains des travaux listés sur ce planning (Planning 27/5-a) sont prévus à l'extérieur. Mais à cause de la pluie, ils ne peuvent être lancés.

Sur cette journée, nous avons repéré 9 cadrages (conduisant à la conception de cadres pour faire, dont 6 cadrages et 3 cristallisations du planning). Ces actions correspondent aux transformations successives de cadres secondaires auxquels le responsable procède seul en s'appuyant sur son planning. Celles-ci sont relatives aux changements météorologiques (alternance de pluie et de soleil) et à des instructions émises par sa hiérarchie (transmission de nouvelles directives). Elles vont nécessiter de la part de l'encadrant de nouvelles spécifications en matière de travaux à lancer.

Nous avons identifié trois cadres secondaires transformés suite à des changements de météo (cadres primaires naturels) : à 8h05 (Cadrage A), 13h20 (Cadrage E) et 14h25 (Cadrage F). Et trois cristallisations ${ }^{9}$ du planning et que nous détaillerons un peu plus loin dans ce paragraphe.

De plus, nous avons constaté deux cadres secondaires transformés suite aux injonctions (cadres primaires sociaux) de sa hiérarchie qui sont émises oralement et au fil de l'eau. Elles sont à l'origine des cadrages $\mathrm{B}$ et $\mathrm{C}$. La première concerne les urgences du matin transmises par le chef de culture et la direction. La seconde est émise en réunion par la direction : « il ne faut pas faire venir les saisonnières à $10 \mathrm{~h}$ car on n'a pas de travail à leur donner». L'encadrant les intercale parmi les travaux en cours et à venir, au coup par coup, développant ainsi son microprojet quotidien.

Un autre élément remarquable réside dans la réorganisation des travaux qui implique de nouvelles spécifications en termes de moyens, par rapport aux effectifs dont il dispose et qui ne sont pas ceux qu'il avait escomptés. Dans un premier temps, parce qu'il a dû reporter

8 Nous reprenons ici le terme de «planning de travaux » utilisé par l'encadrant pour désigner un outil qu'il se construit pour s'aider à penser le travail à faire. Ce planning ne consiste donc pas en une planification de la production à faire qui lui serait transmise par sa hiérarchie, mais plus à un plan au sens de Suchman (1987). Autrement dit, d'une ressource qui lui permet de débuter sa journée en affectant les ouvriers aux différents travaux qu'il doit lancer.

9 Nous appellerons « cristallisation » une matérialisation des cadres secondaires produits par l'encadrant par une action de cadrage. 
l'heure d'arrivée des saisonnières de $10 \mathrm{~h}$ à $13 \mathrm{~h}$. Et dans un second temps, à $13 \mathrm{~h}$ (Cadrage D), car il a été confronté à des absences et des présences imprévues de son personnel. Il avait oublié qu'une saisonnière expérimentée sur laquelle il comptait serait absente cet après-midilà. Et à l'inverse, une saisonnière qu'il n'avait pas appelée pour venir travailler s'est présentée d'elle-même. L'encadrant lui a alors dit de rester même si elle n'avait pas les compétences de sa collègue absente pour la remplacer.

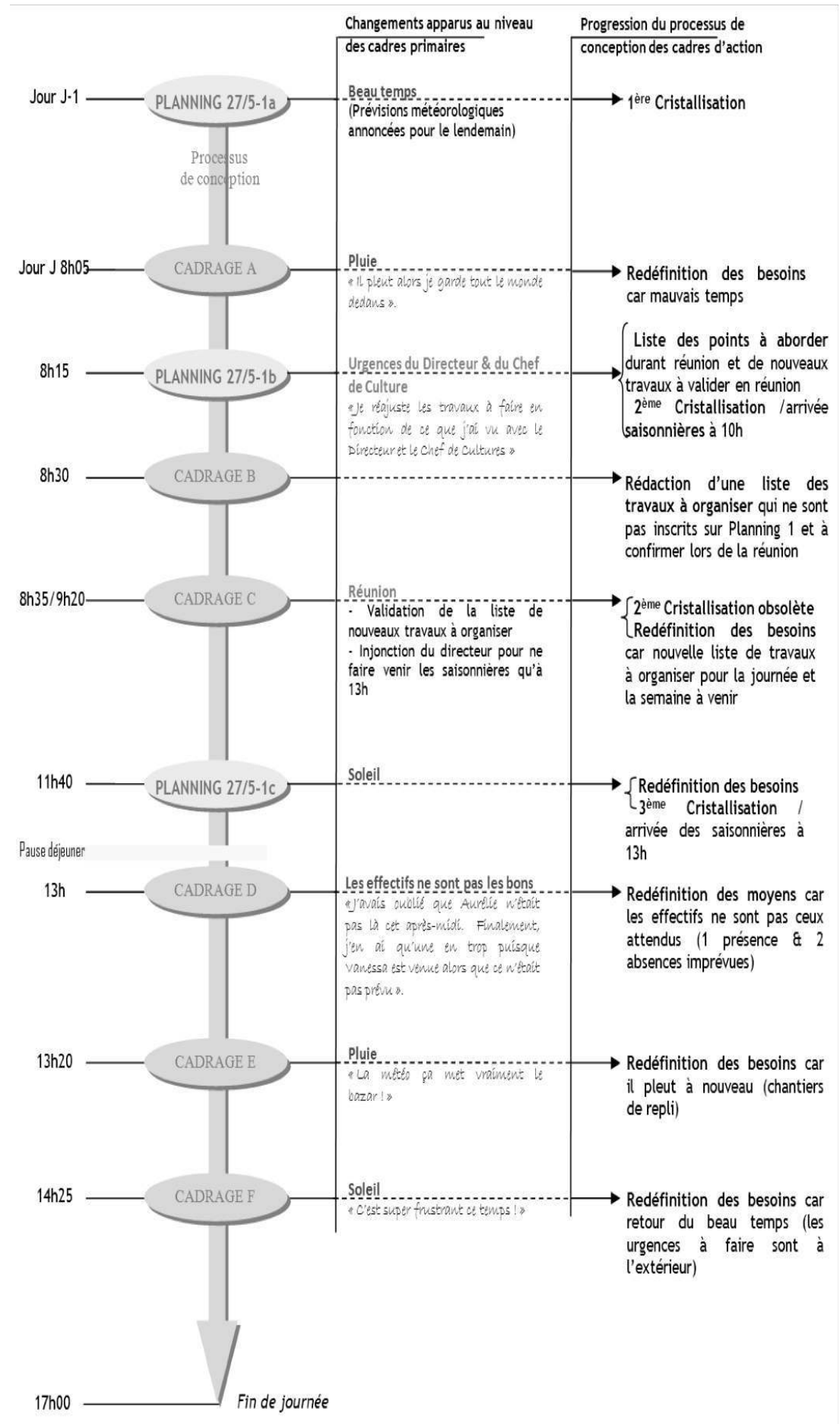

Figure 2 : Le microprojet quotidien conduit par des cadrages successifs

Figure 2: Everyday microproject managed through successive framings

Les trois cristallisations du planning identifiées durant cette journée de travail correspondent à différentes heures d'arrivée des personnels. La première cristallisation (Planning 27/5-a) a 
lieu à $8 \mathrm{~h}$. Elle coïncide avec celle des permanents et de quelques saisonnières expérimentées. La seconde cristallisation (Planning 27/5-b) apparaît juste avant la réunion de 8 h35. À ce moment-là, l'encadrant finalise son planning et détermine les travaux à lancer pour la seconde partie de la matinée, pour l'arrivée des saisonnières prévue à 10h. Mais cette version du planning va s'avérer rapidement obsolète, car durant la réunion et à cause du manque de travail lié à la pluie, la direction lui adresse l'injonction de faire venir les saisonnières ce matin. La troisième cristallisation (Planning 27/5-c) se produit à $11 \mathrm{~h} 40$, alors que la météo est en train de s'améliorer. L'encadrant procède alors à un dernier cadrage pour prévoir les travaux à lancer en vue de l'arrivée des saisonnières pour $13 \mathrm{~h}$.

Cette succession de cadrages (cristallisés ou non dans un planning) témoigne du processus de conception à l'œuvre dans ce microprojet quotidien et conduit par l'encadrant en interprétant les changements survenus dans la situation, au niveau des cadres primaires naturels et sociaux. Intéressons-nous à présent en détail à un microprojet occasionnel.

\section{4.- La conception de cadres pour faire et pour faire faire dans un exemple de microprojet occasionnel}

La conception de cadres d'action, si elle est du ressort de l'encadrant, ne se fait pas isolément. Comme nous l'avons déjà mentionné, les collègues, les subalternes, etc. prennent part à cette conception dans le cadre des microprojets occasionnels.

Dans l'exemple qui suit, le microprojet occasionnel vise à organiser puis à réaliser un chantier de préparation d'une commande de $\mathrm{Phlox}^{10}$ à expédier pour la semaine suivante (cf. Figure 3: «Le microprojet occasionnel conduit par des co-cadrages successifs », située ciaprès). Cette commande correspond à une «offre promotionnelle»- une promesse de vente pour l'entreprise ; les clients s'étant engagés plusieurs mois à l'avance à acheter ces plantes à une date précise. De plus, cette commande représente de grandes quantités ne pouvant être préparées en une seule journée, d'autant plus que le jour de leur départ, d'autres commandes passées le matin même devront, elles aussi, être traitées et expédiées en fin de journée (fonctionnement habituel des commandes). Ces deux préparations ne pourront donc être menées de front.

Dans ce cas, la mission (projet) pour l'encadrant est de livrer cette commande le jour J, pour satisfaire la demande des clients et éviter de passer à côté de cette action commerciale qui représente un véritable enjeu économique pour l'entreprise (gain financier versus production d'un stock à perte vu les quantités). Pour répondre à l'injonction de sa direction, l'encadrant va conduire ce microprojet sur une semaine avec plusieurs jours non consécutifs.

Notre analyse fait apparaître des actions de «co-cadrages» (que ce soit pour des cadres pour faire ou des cadres pour faire faire) réalisés entre l'encadrant et ses collègues de l'entreprise et du groupement. Celles-ci interviennent à chaque phase de la conduite du microprojet. Pour tendre vers son but final, l'encadrant fait appel aux compétences de ses différents collègues pour spécifier chacun des besoins de son chantier et construire pas à pas les différentes étapes de son projet. Chaque co-cadrage constitue donc une étape dans le développement de ce microprojet dont le point de départ est l'annonce de cette vente promotionnelle.

La phase de définition du microprojet comprend 4 co-cadrages (production de cadres pour faire) qui permettent de définir le but du microprojet (co-cadrage 1, 2 et 4) dont le plus représentatif est le co-cadrage 3. Lors de ce dernier, l'encadrant vise à s'assurer de la faisabilité d'un chantier de cette ampleur. Il échange avec le Responsable du Disponible sur la préparation des Phlox et les critères clients à respecter (fleuri, couleurs variées, par plaque de six pots de 2 litres), puis sur l'organisation du chantier de nettoyage (matériel,

10 Le Phlox est une plante vivace (ces racines vivent au-delà de 2 ans). Cela signifie qu'après avoir été prélevées sur la parcelle, elles sont conditionnées sur place (6 par plaque dans ce cas - préparation), puis les plaques passent dans l'atelier où chaque plante est nettoyée (débarrassée de ses feuilles jaunies et mortes - nettoyage) avant d'être regroupée avec les autres pour être expédiée. 
emplacement...). À partir des éléments construits lors de ces premiers co-cadrages, l'encadrant conçoit un cadre pour faire faire en affectant une préparatrice expérimentée pour effectuer le test de préparation des plaques de Phlox.

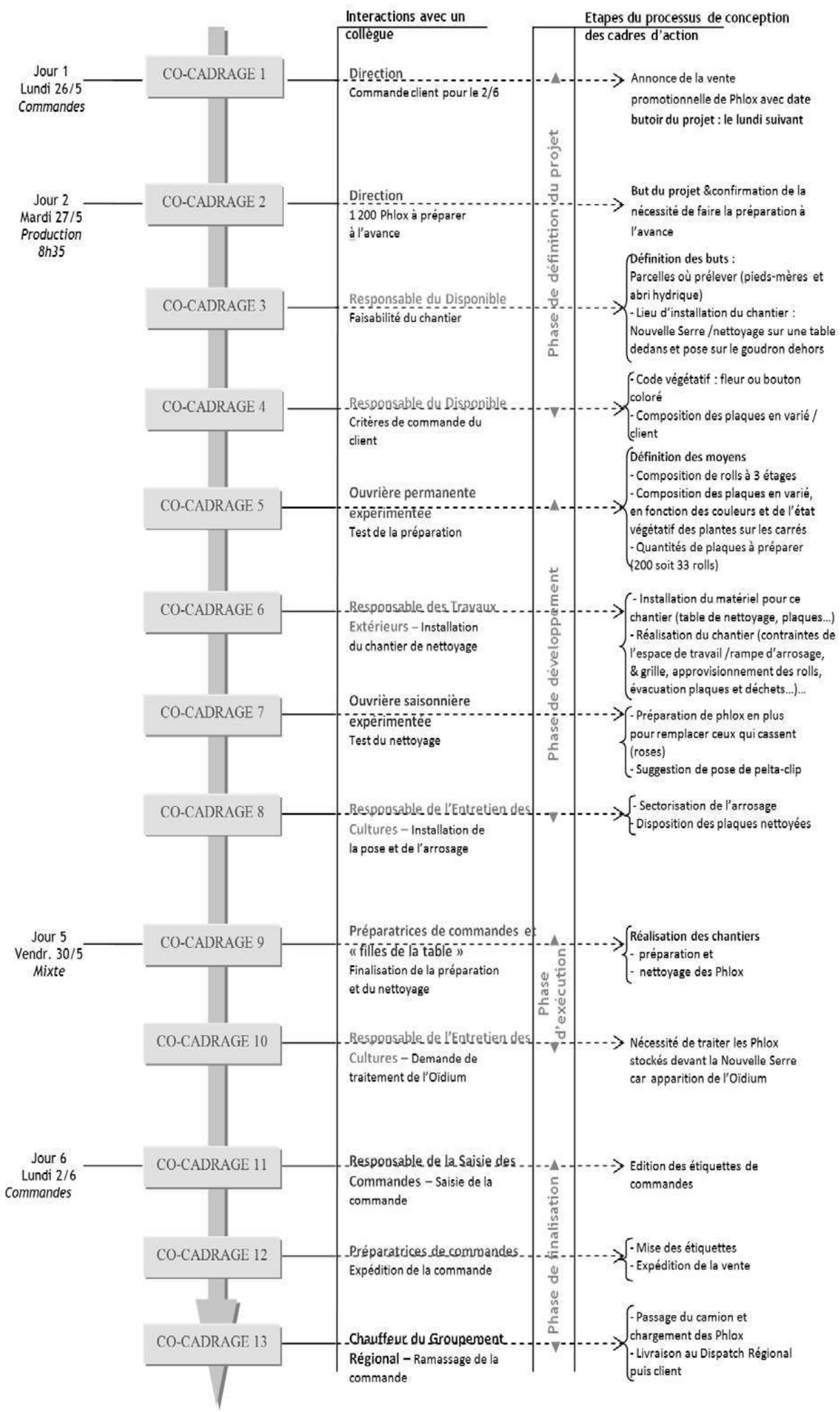

Figure 3 : Le microprojet occasionnel conduit par des co-cadrages successifs

Figure 3: Occasional microproject managed through successive co-framings 
La phase de développement du microprojet se déroule dès le lendemain mardi, en interaction avec des collègues aux compétences spécifiques. Elle comprend 4 co-cadrages dont deux consistent en des tests que nous considérons comme des productions intermédiaires du processus de conception.

— Le co-cadrage 5 se fait avec une permanente expérimentée chargée d'évaluer les possibilités de composition des plaques pour satisfaire aux critères d'exigence des clients. Les données construites par cette préparatrice vont servir à l'encadrant pour concevoir les cadres pour faire faire ensuite cette préparation (et parce qu'il n'en a pas lui-même le temps) aux autres préparatrices qu'il affectera plus tard pour finir le Phlox.

- Le co-cadrage 6: l'encadrant interagit avec la personne qui la seconde en temps de commandes, sur la mise en place du chantier de nettoyage. Celle-ci lui apporte une aide très concrète dans l'aménagement spatial et organisationnel du chantier : ils installent le matériel nécessaire (table, plaques, terreau...) et conçoivent ainsi le «mode opératoire » (cadres pour faire faire) pour les saisonnières qui en assureront la réalisation.

— Le co-cadrage 7 consiste à tester la réalisation de ce chantier avec deux saisonnières expérimentées. Lors de ce test, il ressort que les Phlox roses sont cassants. En conséquence, pour la finalisation de ce chantier, l'encadrant complète son cadre pour faire faire : il ajoute la mise en place de peltaclips sur les pots des plantes nettoyées pour les protéger lors du transport.

— Le co-cadrage 8 renvoie à la collaboration de l'encadrant avec la personne chargée de l'entretien des cultures. Celle-ci est ressource en ce qui concerne l'arrosage des Phlox nettoyés afin de les stocker sur le goudron et pour les conserver dans les meilleures conditions culturales jusqu'à leur expédition.

La phase d'exécution du microprojet a lieu le vendredi suivant et compte 2 co-cadrages :

— Le cocadrage 9 est relatif à la finalisation de la réalisation des deux chantiers par les permanentes (préparation) et les saisonnières (nettoyage). Et parce que le test n'avait pas comme finalité de traiter l'ensemble de la commande.

— Le co-cadrage 10 : l'encadrant constate que parmi les Phlox prêts à être expédiés, certains présentent des tâches d'Oïdium. Afin d'éviter que la maladie ne se propage à l'ensemble du stock durant le weekend, il demande au responsable de l'entretien de cultures de faire un traitement sur l'ensemble de plantes.

La phase de finalisation du microprojet se déroule le jour $\mathbf{J}$ et comprend 3 co-cadrages (11, 12 et 13). Ceux-ci se rapportent respectivement au traitement de la commande pour éditer les étiquettes et les donner aux ouvriers (Co-cadrage 11); à la préparation supplémentaire de Phlox (pour remplacer ceux malades devenus invendables) ainsi que l'expédition de cette vente promotionnelle (Co-cadrage 12); et au départ (dans les temps) de la commande chargée dans le camion (Co-cadrage 13). En effet, le chauffeur du groupement régional passe aux alentours de $16 \mathrm{~h} 30$ sur l'exploitation pour ramasser les commandes de la journée. Après avoir été repartis entre les différentes commandes des clients au dispatch régional, les Phlox seront livrés le lendemain matin aux jardineries.

Dans la conduite de ce microprojet occasionnel, chaque co-cadrage aboutit à la conception d'un cadre pour faire ou pour faire faire. Pour l'encadrant, collaborer avec cette diversité d'interlocuteurs est une manière de s'adjoindre les compétences spécifiques de chacun dont il ne dispose pas personnellement. Cela lui garantit, entre autres, une spécification croissante des connaissances techniques et culturales pour assurer la faisabilité de ce chantier.

Nous constatons aussi l'existence d'irréversibilités comme dans tout projet de conception. Le fait de ne pas traiter les plantes atteintes par la maladie le vendredi comme l'avait demandé l'encadrant, va non seulement mettre en péril toute la commande préparée et prête à partir le jour $\mathbf{J}$, nécessiter une nouvelle préparation dans l'urgence, mais aussi entraîner des avoirretour de la part des clients, et donc avoir des conséquences économiques pour l'entreprise. 
Force est de constater par cet exemple que le fait de travailler avec des produits vivants ajoute une contrainte supplémentaire à gérer dans l'organisation de la production.

\section{5.- Discussion}

\section{1.- Des caractéristiques de l'activité de conception des cadres d'action}

L'activité de conception des cadres d'action est réalisée presqu'exclusivement à l'oral, dans l'action et dans la confrontation à la situation. Elle est structurée par la conduite de plusieurs microprojets quotidiens et occasionnels menés en parallèle. Ce processus aboutit à des transformations successives de cadres secondaires produites par des actions de cadrages (Grossen, 2001) et/ou de co-cadrages, tout au long de la journée de travail, en fonction de l'avancement des travaux. Celui-ci dépend entre autres: des informations transmises au fil de la journée par sa hiérarchie et au gré des échanges avec les collègues, des conditions météorologiques, des évolutions et des urgences du végétal.

Nos résultats montrent le processus de conception comme une stratification (Goffman, 1974) de cadres d'action (ou cadres secondaires transformés). Ces différentes actions permettent à l'encadrant de faire évoluer ses cadres d'action en en poursuivant la conception dans l'action, de manière à rester en phase avec la situation. Cependant, il ne s'agit pas de voir le processus de conception comme une réaction aux «événements», encore moins de l'interpréter comme de l'opportunisme. Comme dans tout projet de conception, nous avons pu observer des itérations, notamment dans les situations de co-cadrages. Mais vu les temps de développement des microprojets menés de front, oralement et sous forte contrainte de temps, ces itérations ne sont pas aussi nombreuses ni perceptibles que dans des projets de conception au timing moins contraint et aux équipes élargies (Staudenmaïer, 1985).

D'autre part, pour gérer son activité de conception des cadres d'action, l'encadrant interagit avec des interlocuteurs situés à tous les niveaux hiérarchiques dans l'entreprise et avec d'autres responsables externes à celle-ci (i.e. dispatch régional). Ainsi, conduire l'ensemble de ces microprojets requiert de prendre en compte différentes logiques propres à chacun des acteurs (par rapport à leur fonction, leur statut, leurs compétences, etc.), et parfois contradictoires.

Cette activité produit des objets intermédiaires témoignant du processus de conception et qui regroupe d'un côté des artefacts pour organiser le travail; c'est le cas des plannings des travaux qui ne sont pas systématiquement matérialisés (ne faisant pas l'objet d'une cristallisation) et dont l'usage reste exclusivement personnel puisque l'encadrant ne les partage avec aucun de ses interlocuteurs. Et de l'autre, des artefacts pour prescrire le travail, comme les consignes transmises essentiellement oralement ou parfois sous forme de notes manuscrites.

En outre, par son activité de conception, l'encadrant opère à une réduction relative de l'incertitude liée qu'à l'élément naturel et à certaines formes de variabilités (humaines et industrielles) demeure un enjeu permanent.

Ainsi, l'activité d'encadrement peut être appréhendée comme une activité de conception complexe développée sous incertitude, à la fois cadrée et cadrante. Elle est cadrée par les cadres primaires (naturels et sociaux) et par la temporalité inhérente aux différents microprojets conduits. Et elle est cadrante par les cadres d'actions qu'elle produit et le lien hiérarchique qu'elle implique.

\section{2.- Une activité cadrée par des cadres pour l'action et ceux de la conception}

Premièrement, les cadres naturels ne sont pas l'œuvre d'une intelligence (Goffman, Ibid.). Or, dans le secteur horticole, l'élément naturel est omniprésent. Il joue donc un rôle 
déterminant pour les personnes chargées d'organiser et de prescrire le travail, car il faut composer avec lui. Ainsi, les transformations se font en continu, en tenant compte des conditions météo et des travaux à faire absolument. Les enjeux économiques et de production sont réels et orientent de manière sous-jacente, mais quasi permanente le travail de l'encadrant. Dans ce contexte, nous ne sommes pas très éloignés des conditions de travail décrites dans le secteur du BTP, avec une production de type «foraine » caractérisée par des situations de travail jamais identiques et dont la production fait évoluer l'espace de travail (Béguin \& Bergamini, 1996 ; Duc, 2002), à la différence que ce n'est pas l'espace de travail qui évolue, mais la nature des travaux (production ou commandes) ainsi que l'état des plantes.

En effet, le travail de production et de commandes est tributaire des conditions météorologiques et des évolutions des produits vivants. L'enjeu pour l'encadrant est de réussir à les prendre en compte pour éviter de mettre en péril la production, tout en garantissant les quantités et la qualité ainsi que la vente des végétaux en temps et en heure, voire avant que certains ne dépassent leur stade de floraison. Car certaines vivaces ne fleurissent qu'une fois l'an. Si elles partent trop avancées ou fanées chez le client, elles risquent d'être retournées à l'entreprise. Cela représente alors une double perte financière pour l'exploitation : leur coût de production et celui de l'avoir-retour réclamé de droit par le client. Dans ce sens, le rôle de l'encadrement est bien déterminant dans la performance de l'entreprise (Mascia, 1994).

Deuxièmement, les cadres sociaux présents dans cette entreprise découlent en partie de l'organisation $\mathrm{du}$ groupement. Cette configuration a pour effet de démultiplier les interlocuteurs externes, de produire des cadres pour l'action élaborée à différents niveaux (national, régional et local). Nous retrouvons cette notion de prescription interne et externe adressée aux conducteurs de travaux (Six \& Tracz, 1997), les confrontant à une multitude de logiques et de prescriptions avec lesquelles ils vont devoir faire des compromis pour réussir à gérer leur activité d'encadrement.

Mais les cadres sociaux renvoient aussi à la variabilité « industrielle » que l'encadrement est amené à réguler et qui a été relevée dans plusieurs études (Bellies \& Beauguil, 2008 ; Lebris, 2010 ; Mascia, 2001). Et dans cette entreprise horticole, malgré les cadres pour l'action et les échanges avec les collègues, l'encadrant doit construire des cadres d'action pour faire et faire faire le travail. Il a émergé de nos résultats qu'une partie du travail de planification des travaux de production émanant du chef de culture n'était pas préparée en amont par ce dernier. Nous assistons à la délégation partielle de ce travail (Dujarier, 2008) de planification à l'encadrant de proximité qui ne disposait pas de toutes les compétences ni de toutes les connaissances culturales pour assurer ce travail pourtant essentiel pour pouvoir en aval exercer son activité d'encadrement. Par conséquent, pris par des contraintes de temps et de charge de travail, il arrive que par manque de temps il les fasse concevoir par des permanents expérimentés avant de les valider, ou encore qu'il les co-construise avec ces mêmes ouvriers.

Troisièmement, l'activité de conception est cadrée temporellement entre son départ et son aboutissement. Tout projet de conception a sa propre temporalité qui compose une contrainte déterminante pour le processus de conception (Midler, 1996). Et cela est valable quelle que soit sa durée (de quelques jours à quelques minutes). Néanmoins, en fonction du temps dont le concepteur dispose, les possibilités ou les opportunités de développer son (micro) projet ne seront pas les mêmes. Ainsi, dans sa pratique, les phases du processus de conception sont extrêmement réduites temporellement et conduites dans la confrontation à la réalité de la situation et de l'accomplissement du travail de production. Dans l'urgence, il arrive que les échanges avec les interlocuteurs prenant part au microprojet occasionnent des tensions ne facilitant pas toujours la construction de la solution ni la poursuite de l'organisation de la production (Béguin, 2004). Sachant que dans ce cas, l'encadrant reprend à sa charge des 
tâches prescrites quand il juge que les conditions de travail ne permettent pas aux ouvriers de faire un travail de qualité, car il ne veut pas «qu'ils s'habituent à faire du mauvais travail ».

L'encadrant de cette entreprise horticole mène plusieurs projets de conception en parallèle. Il les «tient» tous en même temps, passant de l'un à l'autre en fonction des travaux qu'il lance. Les temporalités qu'il a à gérer dans son activité d'encadrement sont donc multiples. Elles ne sont pas sans évoquer celles rapportées par Zara-Meylan (2012) concernant l'activité des chefs de cultures. D'autant plus qu'au travers de notre analyse, nous venons d'évoquer uniquement la facette de son activité relative aux tâches de gestion de la production. De ce fait, à celles-ci s'ajoutent des tâches de gestion des ressources humaines, des tâches commerciales et relationnelles (Mascia, 1994) qui constituent les autres facettes de son travail d'encadrant.

Malgré les cadres pour l'action que nous venons d'évoquer, nos résultats rejoignent ceux des études déjà menées et faisant état de la faible prescription adressée à cette catégorie de travailleurs (Rogard \& Béguin, 1997). Au sein de cette entreprise horticole, l'encadrant dispose de peu d'éléments construits en amont au niveau de l'organisation, définissant ou décrivant les tâches multiples qu'il lui incombe. Il n'existe d'ailleurs aucune fiche de poste dans l'entreprise. Les attendus de son poste se résument à cette injonction de sa direction: «je te paie pour que ça roule »... Reste à savoir ce qu'il est sensé mettre derrière ces termes (Castejon \& Jean, 1998). Concernant la fiche de travaux hebdomadaires qui lui est transmise par le chef de culture: celle-ci lui indique les travaux à lancer dans la semaine pour entretenir les cultures, mais elle ne lui fournit aucune spécification technique culturale lui permettant de mettre en œuvre leur réalisation. L'encadrant doit donc la retravailler pour déterminer le travail à faire et comment le faire faire (Six \& Tracz, 1997).

\section{3.- Une activité cadrante par les cadres d'action conçus et les liens hiérarchiques existants}

Par ailleurs, cette activité est cadrante. Plus précisément, même si une partie consiste en de la transmission telle quelle et à l'adaptation de cadres pour l'action, cette activité d'encadrement productif permet d'aboutir à la conception des cadres d'action au sein de microprojets aux empans temporels variés.

Dans un premier temps, l'objectif du travail de l'encadrant est d'élaborer des cadres ou de définir des champs d'action pour lui-même et pour les autres. Et dans un second temps, il s'agit de s'assurer que les actions déclenchées permettent de réussir à faire le travail demandé. Mais ces cadres ne sont pas tous posés au préalable ni construits en amont de son activité de l'encadrant. Nous l'avons vu, certains sont conçus dans l'action et dans la confrontation au réel de la situation.

Nous avons défini le terme de «cadres pour faire » pour désigner les cadres qu'il construit pour déterminer les travaux à organiser pour lui-même et pour les autres. Dans ce sens, il participe bien à la transformation de l'organisation (Carballeda, 1997a) et même mieux : il crée de l'organisation pour la production. Dans le contexte conception, l'encadrant ne replanifie pas au sens où l'entend Mascia (2001), puisque rien n'est formalisé en amont au niveau de l'organisation de la production. Nous ne pouvons donc soutenir ici que l'activité d'encadrement productif, tel que nous l'entendons, consiste à «faire cadrer » des actions envisagées en amont (de Terssac \& Cambon, 1998). Les cadres secondaires que l'encadrant transforme sont remis en mouvement du fait la contingence de la situation et des changements et se produisant au niveau des cadres primaires naturels et sociaux. Ainsi, de notre point de vue, l'activité d'encadrement dépasse le «faire cadrer» pour le «faire exister » en fonction de la réalité rencontrée.

Dans cette petite entreprise, ce travail de production de cadres se construit à l'initiative de l'encadrant qui est en prise en continu, tout au long de la journée avec la réalité du terrain et qui agit en fonction de l'avancée du travail. Le collectif d'encadrants se compose de quatre 
personnes et dont deux sont les co-gérants (directeur et responsable du disponible), le quatrième étant le chef de culture. Ces personnes sont proches géographiquement et hiérarchiquement. Pour agir, elles sont extrêmement contraintes temporellement (par les composantes inhérentes à la situation) : les actions prescrites sont réalisées dans la foulée, quasi immédiatement après leur conception. Ces moments de conception peuvent être vus comme autant d'espaces de régulations établis conjointement, dans l'action, avec les subordonnés et parfois la hiérarchie (Carballeda, 1997a).

Concernant les cadres pour faire faire, ceux-ci permettent de répondre à une autre question : comment procéder pour réaliser tel chantier ou telle commande? Dans cette phase de l'élaboration de cadres d'action, l'encadrant est en interaction avec ses subordonnés. Au fil du temps, de par son expérience, il s'est construit des principes organisateurs et s'appuie d'une part sur des indicateurs qu'il prélève sur la situation pour savoir comment opérer, et d'autre part sur les savoir-faire des personnes qu'il encadre. Dans ce sens, son expérience, mais aussi celles de ses subordonnés, est déterminante pour faire face au réel de la situation (Pastré, 2005). Il procède à la conception de ces cadres en s'appuyant sur les compétences des ouvriers expérimentés (permanents ou saisonniers), en fonction des différentes tâches à réaliser.

Par ailleurs, il existe un lien de subordination entre les individus en présence. Dans le cas de la conception des cadres d'action, nous avons vu qu'il peut s'agir de l'encadrant et d'un de ses subordonnés, mais aussi de l'encadrant et d'un de ses supérieurs hiérarchiques, voire parfois des trois en même temps. Il semble qu'il y ait des échanges autour de l'action à mener. Néanmoins, nous avons observé que c'est toujours le supérieur hiérarchique qui valide in fine le cadre d'action conçu. Cela est valable aussi dans l'autre sens, c'est-à-dire quand le microprojet met en présence l'encadrant et un des ses supérieurs hiérarchiques : c'est alors à ces derniers que revient la décision finale.

\section{7.- Conclusion}

L'encadrement de proximité, de par sa fonction et son statut, est plus qu'un simple concepteur. C'est un véritable chef de projet. Il est semblable à un chef d'orchestre dans le sens qu'il articule le travail de toute une équipe composée de compétences multiples. Son travail ne consiste pas seulement à faire appliquer des cadres pour l'action élaborés en amont, mais de produire des cadres d'action pour faire et faire faire, en poursuivant leur conception dans l'action avec ces collègues de tous niveaux hiérarchiques. Il assure cette concrétisation tout en prenant en compte d'un côté, la variabilité inter et intra individuelle et de l'autre, la variabilité industrielle inhérente à la situation et dont l'élément naturel accentue la dimension dynamique.

Par conséquent, son activité d'encadrement dépasse la simple définition d'un cadre posé au préalable et par rapport auquel il n'aurait ensuite qu'à s'assure de faire cadrer les actions de ses subordonnés, car il s'agit pour lui de concevoir ses cadres dans l'action, à un moment « $\mathrm{t}$ » en interaction avec ses collègues. Cela nécessite de traiter de l'information parvenant de l'intérieur comme de l'extérieur de l'entreprise, de multiples pratiques, des compétences différentes et des logiques parfois contradictoires, pour faire face aux évènements émergeant de la réalité de la situation. L'activité d'encadrement productif, c'est donc produire avec d'autres pour concrétiser le travail à faire et à faire faire.

La production de connaissances issue de cette recherche est focalisée sur l'activité d'encadrement productif, représentative de la singularité d'une situation et les résultats produits nous semblent opérationnels pour analyser une partie de l'activité des encadrants de proximité, même s'il s'avérerait nécessaire d'observer cette catégorie de travailleurs sur d'autres terrains pour consolider nos résultats. Néanmoins, précisons que notre étude a fait partie d'un projet de recherche européen ayant bénéficié de plusieurs autres terrains 
horticoles qui ont été investigués au sein de notre équipe d'ergonomes. Les travaux menés en parallèle par nos collègues, notamment sur les chefs de cultures de cinq entreprises horticoles, sont venus confirmer les résultats de notre recherche. Ils font d'ailleurs l'objet d'articles communs à venir.

\section{Bibliographie}

Becker, S. (1963). Outsiders, Étude de sociologie de la déviance. Métailié, 1985, p. 35.

Béguin, P. (2004). L’ergonome, acteur de la conception. In P. Falzon (Ed.), Ergonomie (pp. 374-390). Paris: PUF.

Béguin, P. (2010). Conduite de projet et fabrication collective du travail : une approche développementale. Document pour 1'Habilitation à Diriger des Recherches, Université Victor Segalen Bordeaux 2.

Béguin, P., \& Bergamini, J.F. (1996). Organiser la conception pour le chantier. In R. Patesson (Ed.) Intervenir par l'ergonomie. Actes du XXXİ̀me Congrès de la Self, Volume 1 (pp. 219-223). Bruxelles.

Béguin, P., \& Weill-Fassina, A. (1997). La simulation en Ergonomie. Connaître, agir, interagir. Toulouse: Octarès.

Bergamini J.F. (1995). Du virtuel au réel - Quelques aspects de l'activité de chef de chantier. Unpublished Mémoire de DEA d'Ergonomie. Paris, CNAM - EPHE.

Bellies, L. \& Beauguil, L. (2008). L'accompagnement des projets de conception avec et sans objet intermédiaire : conséquence sur la coopération avec les acteurs de la conception. Concevoir pour l'activité humaine. $43^{\text {ème }}$ Congrès de la SELF, Ajaccio, 17-19 septembre. Ajaccio: ANACT, pp. 244-252.

Böhler, D. (1985). Rekonstrucktive Pragmatik. Von der Bewussteinsphilosophie zur Kommunikationsreflexion. Neubegründung der praktishen Wissenschaften und Philosophie, Francfort-sur-le-Main.

Carballeda, G. (1997a). La contribution des ergonomes à l'analyse et à la transformation de l'organisation du travail : l'exemple d'une intervention relative à la maintenance dans une industrie de process continu. Unpublished Thèse de Doctorat d'ergonomie. Paris: CNAM, Laboratoire d'Ergonomie.

Carballeda G. (1997b). Les cadres : des travailleurs en difficulté. Performances Humaines \& Techniques, 91, 11-15.

Carballeda, G., \& Garrigou, A. (2001). Derrière le stress, un travail sans contrainte. In P. Bouffartigues (Ed.), Cadres : la grande rupture (pp. 89-108). Paris: La Découverte.

Castejon, C., \& Jean, R. (1998). Travail et encadrement : quel usage de soi pour quel usage des autres? Performances Humaines \& Techniques, HS Septembre, 28-31.

Detchessahar, M. (2011). Santé au travail. Revue française de gestion, 2011/5(214), 89-105. http://www.cairn.info/revue-francaise-de-gestion-2011-5-page-89.htm

Detchessahar, M. (2013). Faire face aux risques psycho-sociaux : quelques éléments d'un management par la discussion. Négociations, 2013/1(19), 57-80. http://www.cairn.info/revue-negociations2013-1-page-57.htm

Dieumegard, G., Saury, J., \& Durand, M. (2004). L'organisation de son propre travail : une étude en cours d'action des cadres de l'industrie. Le Travail Humain, 67(2), 157-179.

Duc, M. (2002). Le travail en chantier. Toulouse: Octarès.

Dujarier, A-M. (2008). « Prendre sur soi » : l'individualisation du travail d'organisation. In G. Terssac (de), C. Saint-Martin, \& C. Thébault, (Eds.). La précarité : une relation entre travail, organisation et santé (pp. 107-117). Toulouse: Octarès.

Forrierre, J., Anceaux, F., Cegara, J., \& Six, F. (2011). L'activité des conducteurs de travaux sur les chantiers de construction : ordonnancement et supervision d'une situation dynamique. Le Travail Humain, 74(3), 283-308.

Gillet, A. (2003). Transformations professionnelles de l'encadrement de proximité : entre management et expertise technique. Actes de la journée du 8 décembre 2003, les Cahiers du GRD 
Cadres, pp. 55-71.

Goffman, E. (1974). Les cadres de l'expérience. Paris: Les éditions de Midi.

Gotteland-Agostini, C. (2013). Concevoir des cadres pour agir et faire agir : l'activité de prescription dans une entreprise horticole. Unpublished Thèse de doctorat en Ergonomie, CNAM-CREAPT, Paris.

Grossen, M. (2001). La notion de contexte : quelle définition pour quelle psychologie ? Un essai de mise au point. In J.P. Bernier (Ed.), Apprentissage, développement et significations : hommage à Michèle Brossard (pp. 59-76). Presse Universitaire de Bordeaux.

Joas, H. (2004). La créativité de l'agir. Paris: Édition du Cerf.

Langa, P. (1994). Adaptation ou création de l'organisation du travail lors d'un transfert de technologie - Analyse de l'activité de l'encadrement et conception de l'organisation. Unpublished Thèse de Doctorat en Ergonomie, CNAM Paris.

Lebris, V. (2010). La continuité des activités de production dans des systèmes postés discontinus : approche ergonomique des activités de relève de poste de l'encadrement de proximité. Unpublished Thèse de Doctorat en Ergonomie, Université Toulouse le Mirail.

Létondal, A.M. (1997). L'encadrement de proximité. Quels rôles dans les changements d'organisation? Lyon: ANACT.

Mascia, F.L. (1994). La gestion de la production, une approche ergonomique du travail du chef d'atelier. Unpublished Mémoire de D.E.A. d'Ergonomie. Paris: Laboratoire d'Ergonomie Physiologique et Cognitives, EPHE.

Mascia, F.L. (2001). Gérer dans et avec l'atelier : une approche ergonomique du travail de la maîtrise dans le secteur industriel de production à grande échelle. Unpublished Thèse de Doctorat en Ergonomie. Paris: Laboratoire d'Ergonomie Physiologique et Cognitive, EPHE.

Midler, C. (1996). Modèles gestionnaires et régulation économique de la conception. In G. de Terssac, \& E. Friedberg (Eds.), Coopération et conception (pp. 63-85). Toulouse: Octarès.

Mispelblom Beyer, F. (1996). Langages et stratégies au travail saisis par les normes d'assurance qualité. Sociologie du travail, XLI(3), 235-254.

Mispelblom Beyer, F. (2003). Stratégies de direction et dispositifs de management. In Sociologie des dispositifs de gestion. Paris: L'Harmattan.

Mollo, V. (2004) Auto- and confrontation as tools for reflective activities. Applied Ergonomics, 35(6), 531-540.

Nizet \& Rigaux, (2005). La sociologie de Erwing Goffman. Paris: La Découverte.

Pastré, P. (2005). Génèse et identité. In Rabardel, P., \& Pastré, P. (Eds). Modèles du sujet pour la conception, dialectiques activités et développements (pp. 231-260). Toulouse: Octarès.

Petit, J. (2005). Organiser la continuité du service : intervention sur l'organisation d'une mutuelle de santé. Thèse d'Ergonomie. Université Victor Segalen Bordeaux 2- ISPED. Laboratoire d'Ergonomie des Systèmes Complexes.

Piney, C., Nascimento, A., Gaudart, C., \& Volkoff, S. (2013). Entre indicateurs et travail réel : l'expérience de l'encadrement de proximité dans un service public. Actes du $48^{\text {ème }}$ congrès, et $50^{\text {ème }}$ anniversaire, de la Self, Ergonomies et société : quelles questions, quelles réponses ?, 28-30 août, La Sorbonne, Paris.

Schön, D.A. (1983). The reflexive practitioner: how professionals think in action. USA: Basic Books.

Rogard, V., \& Béguin P. (1997). L'ergonomie au risque du travail des cadres. Performances Humaines \& Techniques, 91, 8-10.

Six, F. (1999). De la prescription à la préparation du travail : apports de l'ergonomie à la prévention et à l'organisation du travail sur les chantiers de bâtiment. Document pour l'Habilitation à Diriger des Recherches, Lille: Université Charles de Gaulle Lille 3.

Six, F., \& Tracz, C. (1997). L'encadrement de chantier : des évolutions sous le regard de l'ergonomie. Performances Humaines \& Techniques, 91, 25-29.

Sharron, A. (1981/2000). Frame paralysis: when time stands still. In G.A. Fine, \& G.W.H. Smith (Eds.), Erwing Goffman, Sage Publicashion, Sage masters of modern social thoughs (pp. 94-108). London: Thousnad Oasks. 
Staudenmaïer, J.M. (1985). Technology's Strorytellers. Cambridge, Massachusetts: MIT Press.

Suchman, L. (1987). Plans and situated actions: the problem of human, machine interaction. Cambridge: Cambridge University Press.

Terssac, G. de, \& Cambon, P. (1998). Le travail d'encadrement : relation entre cadre et action. Performances Humaines \& Techniques, HS Septembre, 49-53.

Van Belleghem, L. (2013). Réciprocité des enjeux de confiance au travail - Le cas des coursiers et de leur dispatcheur. In L. Karsenty (Ed.), La confiance au travail (pp. 53-75).Toulouse: Octarès.

Wolff, L. (2013). Un encadrement de plus en plus... encadré. Santé et Travail, N82, avril, 30-32.

Zara-Meylan, V. (2012). Modalités de gestion du milieu temporel dans une conduite de processus multiples en situation dynamique : une recherche dans des entreprises horticoles. Unpublished Thèse de doctorat en Ergonomie, CNAM-CREAPT, Paris.

\section{RESUME}

Ce texte a pour but de produire des connaissances sur une partie du travail des encadrants concernant tout particulièrement la gestion de la production : leur activité d'encadrement productif. Nous considérons cette activité comme une activité de conception qui produit des cadres de travail (pour faire et faire faire le travail) destinés à lui-même et aux autres. Pour cela, nous nous appuyons sur une recherche menée dans une petite entreprise horticole appartenant à un groupement national de producteurs, lui-même composé de trois groupements régionaux. Ce groupement définit en partie le fonctionnement interne de l'entreprise. Il impose des cadres qui préexistent au travail de l'encadrement ainsi que des interlocuteurs externes à l'entreprise avec lesquels l'encadrant se doit d'interagir. L'analyse des observations et des entretiens montre que cette activité d'encadrement productif se développe par la conduite de microprojets quotidiens et occasionnels. L'encadrant procède seul ou avec l'aide de ses collègues. Avant de prescrire, il doit organiser la production. Cela se fait dans la confrontation à la situation de travail qui évolue en permanence. Il apparaît alors comme étant bien plus qu'un simple concepteur dont l'activité est à la fois cadrée et cadrante. Il est en effet un véritable chef de projet chargé d'articuler le travail de toute une équipe aux compétences multiples, dont il s'agit de coordonner le travail pour atteindre les objectifs de production et de vente.

\section{MOTS CLEFS}

encadrant, encadrement, management de la production, cadre d'action, microprojet

\section{REFERENCEMENT}

Gotteland-Agostini, C., Pueyo, V., \& Béguin, P. (2015). Concevoir des cadres pour faire et faire faire : 1' activité d' encadrement dans une entreprise horticole. Activités, 12(1), 24-45. http://www .activites.org/v12n1/v12n1.pdf

Article soumis le 02/07/2014, accepté le 02/01/2015 